\title{
Toward the Design of Socio-Technical Systems for Sustainability: A Scientometric Analysis
}

\author{
Zijia Wang ${ }^{1,2}$ Han-Teng Liao ${ }^{1,2, *}{\text { Yujin } \text { Zhou }^{2} \text { Sixia Pang }}^{3}$
}

\author{
${ }^{I}$ New Media Research Centre, Sun Yat-sen University Nanfang College, Guangzhou, Guangdong, 510970, China \\ ${ }^{2}$ School of Literatures and Communications, Sun Yat-sen University Nanfang College, Guangzhou, Guangdong, 510970, China \\ ${ }^{3}$ College of Attainment, Sun Yat-sen University Nanfang College, Guangzhou, Guangdong, 510970, China \\ *Corresponding author. Email: h.liao@oxon.org
}

\begin{abstract}
Major challenges facing humanity today require concerted efforts in using technologies for systemic change and the concept of socio-technical system has been used by multiple disciplines to understand the complex interactions of technologies and human behaviors. The paper aims to map out the main sources, disciplines, and keywords of the literature, exploring how it has grown out of its roots in industrial sociology and the Human Relations Movement. Based on 1,968 articles collected from the Web of Science (WoS) database, the findings show that socio-technical system research has involved several disciplines, including the two main discipline clusters of (a) ergonomics, safety science \& engineering and (b) sustainability science \& applied social studies of science. Also, the main research keywords include design (related to product, production, interaction, cognitive work analysis, and information systems), complexity (related to human factors, safety, resilience, risk and health care), transition (related to innovation, sustainability, governance, and multi-level perspective), and technology (related to Internet, behavior, consumption, and ethics). The scientometric analysis shows the central role of design for systemic change in the research fronts of socio-technical systems. Keywords: user interaction, systemic change, sustainability, production, consumption, design
\end{abstract}

\section{INTRODUCTION}

Previously used by work organization researchers as a guiding concept to conduct action research to examine the "structure of autonomous work groups, matrices and networks" as the "building blocks for organization design"[1], the notion of "socio-technical systems"(STS) have been developed from its roots in industrial sociology and the Human Relations Movement[2] to areas such as sustainability studies and Human-computer interaction (HCI). It has led to the development of the notion of "sociotechnical transition" towards sustainability [3]. Also the HCI research addresses the relationship between people and Information Technologies (IT), while the STS projects tackle the more-complex and higher-level relationship between communities and $\mathrm{HCI}[4,5]$.

As our social systems have undergone the digitalization process[6], the STS approach may provide a needed perspective, for designers, social science scholars, engineers, etc. to better understand the impact of technical change in the areas of digitalization of finance for sustainable development [7,8], eco-design of Internet [9], the restructuring life and livelihoods during a pandemic such as COVID-19, etc. However, little has been done recently to systematically review the research outcomes of
STS. The paper aims to fill such a gap by providing an upto-date scientometric analysis on the topic.

\section{DATA AND METHODS}

On STS, the paper used the advanced search query below to collect data from the Web of Science (WoS) database:

- $\quad \mathrm{TS}=($ "Socio-technical system*" OR "Socio*technical system*")

1,968 articles were collected on January 24, 2020 (including SCI-EXPANDED, SSCI, A\&HCI, ESCI.).

In addition to our descriptive scientometric analysis, cocitation, bibliographic coupling, and co-word analysis (based on clustering) were conducted to identify current research fronts $[10,11]$, i.e. special domains compose of main keywords, sources and the associated disciplines. VOS viewer was used for clustering and visualizing results.

\section{RESEARCH FINDINGS}

Figure 1 shows that the annual publication counts have been risen rapidly since 2011, indicating increasing interests in research related to STS. To explore how STS research has evolved and stayed relevant, the following findings have been presented, covering main clusters of publication 
sources, disciplines, keywords, and the significant relationship between sources and topics.

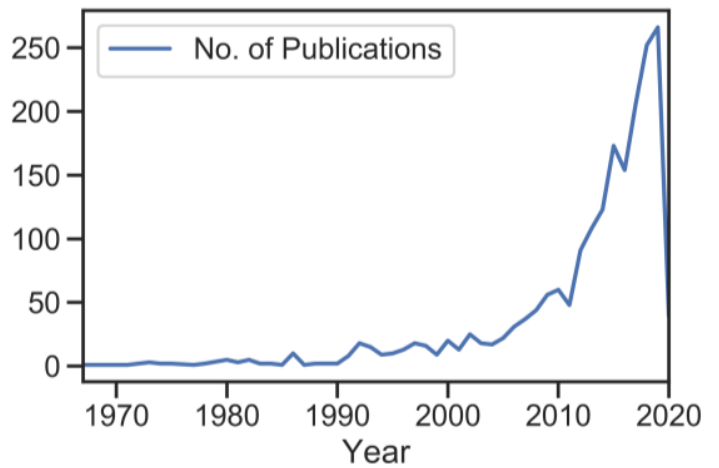

Figure 1 The number of publications

\subsection{Main clusters of sources and disciplines}

With the aim to reveal main clusters of sources (i.e. publication outlets) and disciplines (i.e. the discipline categories of such sources), Figure 2 and Figure 3 first shows the two perspectives of sources. Altogether, they constitute parts of the research fronts [11] to be analyzed.

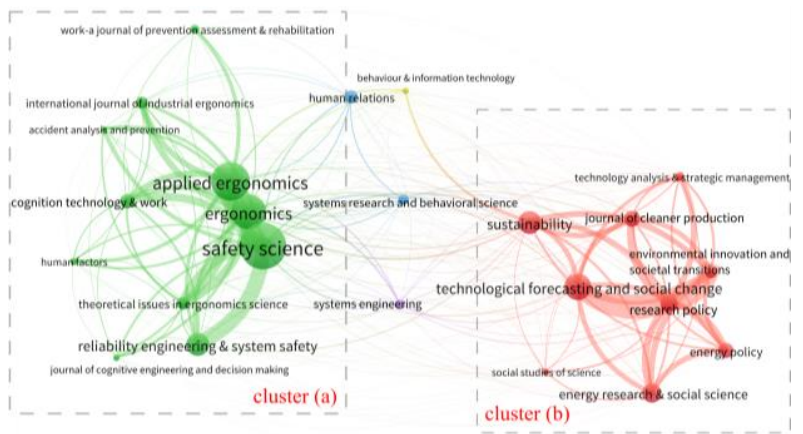

Figure 2 Top publication sources: a bibliographic coupling relationship network visualization

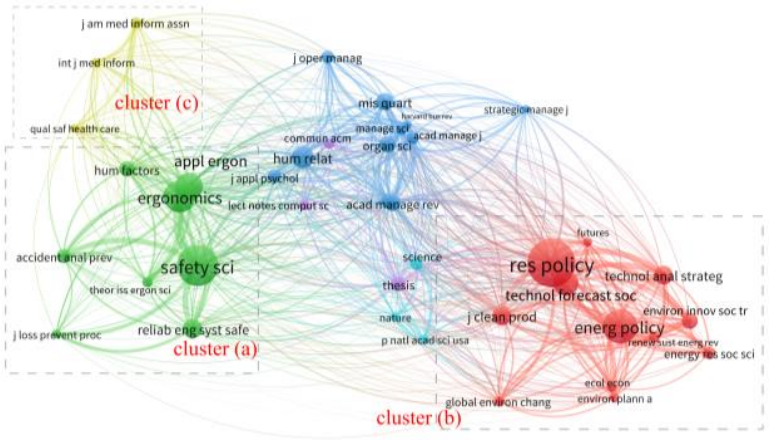

Figure 3 Top cited sources: a co-citation relationship network visualization

\subsubsection{Clusters of publication sources}

Indicating where STS work has been published, Figure 2 indicates how the top two clusters dominate. The cluster at the left shows the top publication sources such as Safety Science, Ergonomics, Applied Ergonomics, etc. The cluster at the right indicates the top sources such as Technological forecasting and Social Change, Sustainability, Energy Research and Social Science, Journal of Cleaner Production, Energy Policy, Research Policy, etc. Based on the disciplines of sources, we name them as follows.

The first cluster (a) "ergonomics, safety science \& engineering" focuses on ergonomics, mainly at the intersections of engineering and psychology.

The second cluster (b) "sustainability science \& applied social studies of science" focuses on sustainability and research policies. It is at the intersections of business, economics, ecology, and science \& technology.

The remaining clusters of sources are situated between the two clusters in Figure 2. The main one is composed of Human Relations and Systems Research and Behavioral Science, clearly indicating the roots of STS in industrial sociology and the Human Relations Movement[2]. The other single-item clusters are Behavior \& Information Technology and Systems Engineering, corresponding to engineering sub-disciplines such as 'computer science' and 'operations research \& management science' respectively.

\subsubsection{Clusters of citing sources}

Indicating where STS work has been citing, Figure 3 indicates how the similar top two clusters dominate.

The cluster (a) at the left shows the mostly similar top publication sources such as Safety Science, Ergonomics, Applied Ergonomics, etc., as shown in Figure 2. The second cluster at the right indicates the top sources such Research Policy and Energy Policy. Note that for the second cluster (b), the top publication sources (see Figure 2) differs from the top-cited sources (see Figure 3). In other words, for this cluster, more STS work cites Research Policy and Energy Policy while more STS work is published in Technological forecasting and Social Change and Sustainability.

The remaining clusters of sources are mostly situated between the two clusters in Figure 3. In addition to thesis, major top science journals (such as Science and Nature), the STS work appears to cite much of Computer Science, Information Science and Management Science literature sources. Also, medicine information and health relation research cluster (c) is shown at the top-left corner of Figure 3 , indicating that although the cluster connects to the main cluster (b) of "ergonomics and safety science \& engineering" and other clusters in the middle, without substantial connections to cluster (a). 


\subsection{Main cluster of author keywords}

To explore how concepts and topics are clustered, Figure 4 shows the co-occurrence network based on top 50 author keywords, which have been . Note that since every article in the dataset contains the term "socio-technical system", it is deliberately omitted in our visualization.

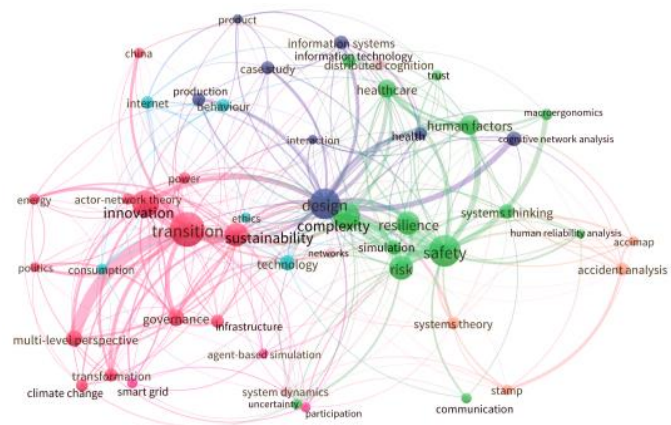

Figure 4 A map based on bibliographic keyword cooccurrence (author keywords)

At the center, the keyword "design" is shown to be related to product, production, interaction, cognitive work analysis, and information systems. Authors have provided keywords in their articles such as "design", "system design", "sociotechnical systems design", "service design", "design for sustainability", etc.

Also, at the center a bit to the right, the "complexity-safetyhuman factors" cluster feature topics such as human factors, safety, resilience, risk, healthcare, macroergonomics, simulation, systems thinking, trust, uncertainty, etc.

At the left, the "transition and sustainability" cluster features keywords such as transition, innovation, sustainability, governance, and multi-level perspective.

Finally, largely overlapping with the "transition and sustainability" cluster, the "internet-technology" cluster contains topics such as behavior, consumption, and ethics.

\subsection{Relationship between sources and topics}

Based on the clusters of (publication) sources, we further explored the corresponding topics (as expressed through author keywords), using the Python scripts we developed. The main source cluster (ergonomics and safety science \& engineering) focuses much more on topics such as safety, resilience, risk, healthcare, macro ergonomics, etc. In contrast, the second cluster (sustainability science and applied social studies of science) focuses more on topics such as transition, innovation, and sustainability.

\section{CONCLUSION}

The scientometric analysis, based on 1,968 articles collected from the WoS database, has resulted in an updated review of socio-technical system literature. It includes two main discipline clusters of (a) ergonomics, safety science \& engineering and (b) sustainability science \& applied social studies of science. The first discipline cluster mostly examines machine and engineering systems in relation to human factors. The second discipline cluster focuses more on the social, knowledge, and even ecological systems.

As shown by our visualization of the author keywords, the body of work largely focuses on the following topics and/or concepts: design (related to product, production, interaction, cognitive work analysis, and information systems), complexity (related to human factors, safety, resilience, risk and health care), transition (related to innovation, sustainability, governance, and multi-level perspective), and technology (related to Internet, behavior, consumption, and ethics). It shows that the impact of technical change has been commonly seen as the complex interactions of technologies and human actions, particularly in both the academic and professional work of HCI, computer science and operations management.

The scientometric analysis shows the central role of design for systemic change in connecting different groups of ideas and authors identified in the research fronts of sociotechnical systems. As email, online chats, social media, collaborative software, etc. have reshaped the way people produce and even consume, we need more sustainable design for socio-technical systems of profits, people and planet. Policy makers, designers, engineers, social scientists, and decision-makers of organizations need conceptual tools to discuss and develop how systemic change is viable by designing both the social and technical components of the digitalization, including the digital design for advancing more sustainable livelihoods, consumption and production.

\section{ACKNOWLEDGMENT}

The research is supported by the Curriculum Development Projects of "API, Machine Learning and AI" (NFU 0240248) "Big Data" (NFU 02-40249) and "Information Visualization Design" (NFU 02-40250), under the Guangdong Department of Education 2018 Grants.

\section{REFERENCES}

[1] E. Trist, The evolution of socio-technical systems: a conceptual framework and an action research program, Ontario Ministry of labour, Toronto, 1981.

[2] J. Scott, Socio-technical system, A Dictionary of Sociology. (2015).

http://www.oxfordreference.com/view/10.1093/acref/97 80199683581.001.0001/acref-9780199683581-e-2207 (accessed January 26, 2020).

[3] F. Geels, Socio-technical transitions and system innovation: Insights from sociology of innovation and evolutionary economics. Keynote presentation IPCC scoping meeting., Geneva, 2016. 
[4] G. Baxter, I. Sommerville, Socio-technical systems: From design methods to systems engineering, Interacting with Computers. 23 (2011) 4-17. https://doi.org/10.1016/j.intcom.2010.07.003.

[5] B. Whitworth, A. Ahmad, 24. Socio-Technical System Design, Encyclopedia of Human Computer Interaction. (2006) 533-541.

[6] E. Tarkhanova, E. Chizhevskaya, N. Baburina, Institutional Changes and Ditigalization of Business Operations in Financial Institutions, Journal of Institutional Studies. 10.0 (2018) 145. https://doi.org/10.17835/2076-6297.2018.10.4.145-155.

[7] UNDFTaskForce, Harnessing Digitalization in Financing of the Sustainable Development Goals, UN Task Force on Digital Financing of the Sustainable Development Goals, 2019.

[8] S. Zadek, The Financial System We Need: Aligning the Financial System with Sustainable Development, IMF/World Bank Group Annual Meetings, Lima, Peru, 2018.

[9] Z. Wang, H.-T. Liao, Towards the eco-design of Artificial Intelligence and Big Data applications: a bibliometric analysis of related research, in: AIBDA 2019, Guangzhou, China, 2019.

[10] E. Garfield, Research fronts, Current Comments. (1994).

[11] O. Persson, The intellectual base and research fronts of JASIS 1986-1990, Journal of the American Society for Information Science. 45 (1994) 31-38. https://doi.org/10.1002/(SICI)1097-

4571(199401)45:1<31::AID-ASI4>3.0.CO;2-G. 